\title{
Huella hídrica del cultivo de aguacate cv. Hass (Persea americana Mill.), en el Distrito de Conservación de Suelos Barbas - Bremen, Quindío, Colombia ${ }^{1}$
}

\section{Water footprint of avocado crop cv. Hass (Persea americana Mill.), at Soil Conservation District Barbas - Bremen, Quindío, Colombia}

\author{
J. F. Naranjo, y H. Reyes
}

Recibido: octubre 13 de 2020 - Aceptado: julio 13 de 2021

\begin{abstract}
Resumen - Se empleó la Huella Hídrica como indicador de la sostenibilidad ambiental de un cultivo de aguacate cv. Hass (Persea americana Mill.) ubicado en la zona sur de la reserva natural denominada Barbas Bremen, bajo la categoría de Distrito de Conservación de Suelos, en jurisdicción del departamento del Quindío. Lo anterior, permitió realizar una descripción general del cultivo, así como determinar los componentes de la huella hídrica verde proveniente de la precipitación $\left(3630 \mathrm{~m}^{3} /\right.$ ton$)$, la huella hídrica azul captada de las fuentes corrientes naturales $\left(0,0 \mathrm{~m}^{3} /\right.$ ton $)$ y finalmente la huella hídrica gris relacionada a la contaminación hídrica (1315 $\mathrm{m}^{3} /$ ton); estimando así, una huella hídrica total de $4.945 \mathrm{~m}^{3}$ /ton correspondiente a dos ciclos de producción de frutos en un año, entre abril de 2019 a 2020 de acuerdo al comportamiento fenológico del cultivo. Así mismo, se formularon algunas recomendaciones tendientes a propiciar una mejor gestión del recurso hídrico en el cultivo aportando al desarrollo ambientalmente sostenible del área de conservación desde la unidad de estudio.
\end{abstract}

Palabras clave - Huella hídrica, Aguacate cv. Hass, Distrito de conservación de suelos, sostenibilidad ambiental.

Abstract - The Water Footprint was used as an indicator of the environmental sustainability of an avocado crop cv. Hass (Persea Americana Mill.) Located in the southern area of the

\footnotetext{
${ }^{1}$ Producto derivado del proyecto de Maestría en Desarrollo Sostenible y Medio Ambiente. "Huella hídrica del cultivo de aguacate cv. Hass (Persea americana Mill.), en el DCS Barbas - Bremen, Quindío, Colombia”, de la Universidad de Manizales.

J.F. Naranjo, Universidad de Manizales, Armenia, Colombia, email: jhonfaber.naranjo@gmail.com.

H. Reyes. Universidad de Manizales, Manizales, Colombia, email: hreyes@umanizales.edu.co.
}

Como citar este artículo: Naranjo, J. F. y Reyes, H. Huella hídrica del cultivo de aguacate cv. Hass (Persea americana Mill.), en el Distrito de Conservación de Suelos Barbas - Bremen, Quindío, Colombia, Entre Ciencia e Ingeniería, vol. 15, no. 29, pp. 63-70, enero-junio 2021.

DOI: https://doi.org/10.31908/19098367.1813.

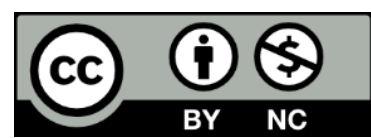

Attribution-NonCommercial 4.0 Intenational (CC By-NC 4.0) nature reserve called Barbas Bremen, under the category of Soil Conservation District, in the jurisdiction of the department of Quindío. The above allowed a general description of the crop to be made, as well as to determine the components of the green water footprint from precipitation $\left(3630 \mathrm{~m}^{3} / \mathrm{ton}\right)$, the blue water footprint captured from natural current sources $\left(0,0 \mathrm{~m}^{3} /\right.$ ton $)$, and finally the gray water footprint corresponding to water pollution $\left(1315 \mathrm{~m}^{3} /\right.$ ton $)$; thus estimating a total water footprint of 4945 $\mathrm{m}^{3} /$ ton corresponding to two cycles of fruit production in one year, between April 2019 and 2020 according to the phenological behavior of the crop. Likewise, some recommendations were formulated to promote better management of the water resource in the crop, contributing to the environmentally sustainable development of the conservation area from the study unit.

Keywords - Water footprint, Avocado cv. Hass, Soil Conservation District, Environmental Sustainability.

\section{NOMENCLATURA}

HH: Huella hídrica

DCS: Distrito de conservación de suelos

BB: Barbas- Bremen

SINAP: Sistema Nacional de Áreas Protegidas

CRQ: Corporación Autónoma Regional del Quindío

Peff: Precipitación efectiva

Kc: Coeficiente de cultivo

ETc: Evapotranspiración de cultivo

ETo: Evapotranspiración de referencia

\section{INTRODUCCIÓN}

LA sostenibilidad de los recursos hídricos en el mundo, $\mathbf{A}_{\text {se }}$ le presenta un desafío importante relacionado con el aumento exponencial de la demanda de agua necesaria para satisfacer las crecientes necesidades de la población, entre ellas la provisión de alimentos en cantidad y de calidad. La agricultura es uno de los pilares fundamentales de la economía colombiana y el sector con mayor participación en el uso del agua ocupando el $43,1 \%$ de la demanda hídrica nacional. Todos los cultivos presentan una leve tendencia creciente, dado que para todos se espera un discreto aumento del área 
sembrada; a excepción de la palma y el aguacate que tendrán un crecimiento considerable y por consiguiente un probable aumento de la demanda de agua [1].

La evaluación del uso del agua durante el proceso de producción agrícola, considerando la variabilidad climática, contribuiría a incentivar una mejor gestión en las prácticas de manejo del agua en un área determinada [2]. Así mismo, las políticas nacionales enfatizan en la necesidad de tecnificar la agricultura para lograr un uso óptimo del agua y aumentar la producción, por tanto, el conocimiento del patrimonio hídrico en cuanto a distribución, estado, dinámica y presiones sobre sus sistemas, constituyen el soporte para la planificación del territorio y la toma de decisiones sobre el mismo [1], [3].

En consecuencia, la huella hídrica se ha propuesto como una herramienta que puede determinar el volumen de agua consumida por unidad de producción, permitiendo estimar los impactos de un proceso antrópico sobre el recurso, respecto a la cantidad y calidad, para lo cual se definen tres componentes fundamentales: la huella hídrica verde, azul y gris. Los dos primeros se relacionan con el impacto en la cantidad de agua y el tercer componente con el impacto sobre la calidad del agua [1], [4], [5], [6] - [7].

También se puede presentar como un indicador de sostenibilidad, ya que ofrece una visión del agua distinta a la convencional, detectando los impactos sobre el recurso hídrico a causa de los hábitos de consumo en actividades socioeconómicas de grupos poblacionales en ubicaciones geográficas específicas [2].

El Quindío se encuentra entre los nueve departamentos colombianos que representan aproximadamente el $86 \%$ del total del área sembrada de aguacate en el país [8], siendo la variedad Hass (Persea americana Mill.) de gran interés en la actualidad del mercado tanto nacional como internacional donde la demanda crece considerablemente [9], [10].

Lo anterior, hace que la ampliación de áreas de siembra para este tipo de cultivo sea inminente, llegando así cada vez con más frecuencia a zonas de fragilidad ecosistémica e importancia ecológica, normalmente sin prever posibles impactos ambientales y afectaciones en la disponibilidad de recursos naturales. Es por esto, que en la actualidad persiste la necesidad de practicar una agricultura más productiva y con un menor nivel de riesgo, la estrategia más clara y precisa es aquella que implica la producción de cultivos en ambientes que provean condiciones que satisfagan los requerimientos agroecológicos de las plantas [11] y que a su vez se desarrollen de manera ambientalmente sostenible.

Esta investigación aborda un tema que no ha sido indagado a profundidad y que busca aportar al manejo de una problemática que se puede acrecentar en los próximos años en la región. Es de anotar que llama la atención la poca información que existe en la literatura nacional e internacional sobre los impactos en el medio ambiente de la producción de aguacate [12].

El estudio tiene como objetivo determinar la huella hídrica de un cultivo de aguacate cv. Hass (Persea americana Mill.) en dos ciclos de producción de frutos, además de analizar comparativamente los resultados obtenidos con tendencias agrícolas nacionales y mundiales bajo la mirada de los impactos antropogénicos en la zona sur de un área de protección ambiental, denominada Distrito de Conservación de suelos Barbas Bremen (DCS BB), donde en la actualidad convergen una alta riqueza biológica y diferentes actividades agroindustriales.

\section{MATERIALES Y MÉTODOS}

\section{A. Área de estudio}

El estudio se realizó en la finca La Florida a una altura a 1.800 msnm (Fig. 2), ubicada en la vereda Membrillal del municipio de Circasia, inmersa en la zona sur de un área categorizada por el Sistema Nacional de Áreas Protegidas (SINAP) como DCS BB (Fig.1), correspondiente a la jurisdicción del Departamento del Quindío [13].

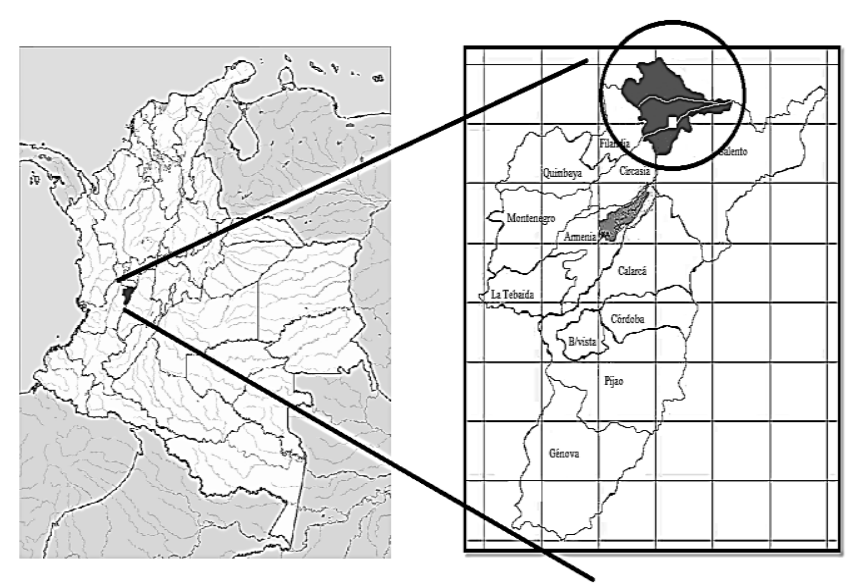

Fig. 1. Localización del área de estudio.

La unidad de estudio se ubica en un piso térmico FrioMedio húmedo y muy húmedo, con coordenadas N 5143'46" - E 42 $90^{\prime} 35^{\prime \prime}$. Posee como base de producción agropecuaria, el cultivo de aguacate cv. Hass (Persea americana Mill.) desarrollado en suelos Francos con buena permeabilidad [13].

\section{B. Diagnóstico general del sistema productivo}

Se determinó el área total sembrada mediante captura y digitalización de imágenes aéreas empleando software libre DroneDeploy, producto de un sobrevuelo no tripulado automatizado de Drone, simultáneamente se recolectó información primaria por medio de la adaptación de guías de caracterización de sistemas agropecuarios [14], [15] de las condiciones del sistema productivo como la descripción general de la unidad productiva, la información climatológica de variables de interés, el aporte de nitrógeno por fertilización al cultivo y finalmente una caracterización del comportamiento general en el tiempo de las etapas fenológicas del cultivo en la unidad de estudio.

C. Cálculo de la huella hídrica en la producción de frutos de aguacate cv. Hass (Persea americana Mill.) en el DCS $B B$

Para el cálculo de la huella hídrica total, se empleó la metodología propuesta por Hoekstra, Chapagain, Aldaya y 
Mekonnen [4], considerando la suma de sus componentes verde, azul y gris, los cuales distinguen las fases del agua que no son enteramente iguales en cuanto a acceso, posibilidad de uso o calidad.

HHcultivo $=\left(m^{2} /\right.$ ton $)=$ HHVerde + HHAzul + HHGris $(1)$

1) Calculo de la huella hídrica verde y azul $\left(H_{\text {Verde }} y\right.$ $\left.\mathrm{HH}_{\text {Azul }}\right)$

Ambos componentes se determinan a partir de las siguientes ecuaciones 2 y 3 :

$$
\begin{aligned}
& \text { HHVerde }=\frac{\text { UACVerde }}{r}\left(\mathrm{~m}^{3} / \text { ton }\right) \\
& \text { HHAzul }=\frac{\text { UACAEul }}{Y}\left(\mathrm{~m}^{3} / \text { ton }\right)
\end{aligned}
$$

Donde $\mathrm{Y}(\mathrm{Ton} / \mathrm{ha})=$ rendimiento del cultivo, $\mathrm{UAC}=$ uso de agua del cultivo verde y azul, según su fuente (precipitación o riego), expresada en $\mathrm{m}^{3} / \mathrm{ha}$ [16].

\section{a. Calculo de uso del agua del cultivo (UAC) $\left(\mathrm{m}^{3} / \mathrm{ha}\right)$}

Se utilizaron las siguientes ecuaciones:

$$
\begin{aligned}
& \text { UACVerde }=10 x \sum_{d=1}^{\lg } \text { ETVerde [Volumen/ärea] } \\
& \text { UACAzul }=10 x \sum_{d=1}^{\mathrm{lgp}} \text { ETAzul [Volumen/ärea] }
\end{aligned}
$$

Donde $\Sigma=$ ciclo de crecimiento del cultivo, es decir, desde el inicio del periodo en estudio (día 1) hasta la cosecha establecida, $\operatorname{lgp}=$ longitud, días del ciclo y ET = evapotranspiración del cultivo ( $\mathrm{mm} / \mathrm{día})$.

- Cálculo de la evapotranspiración (ETo) y determinación del coeficiente de cultivo $(\mathrm{Kc})$

El clima define la demanda evaporativa de la atmósfera, que puede ser determinada a partir de la ETo, siendo en consecuencia independiente de las características de un cultivo particular [17]. Para su cálculo, se han desarrollado diferentes ecuaciones empíricas o semiempíricas, utilizando la información climática [16]. De acuerdo a lo anterior, se utilizó el método de Penman-Monteith por medio del programa Cropwat 8.0 [18]. El Kc describe las variaciones de la cantidad de agua que las plantas extraen del suelo a medida que se van desarrollando, desde la siembra hasta la recolección [19]. Este valor no se conoce para aguacate var. Hass en condiciones edafoclimaticas de Colombia [17].

- Estimación de evapotranspiración del cultivo (ETc).

La ETc se considera igual al requerimiento de agua del cultivo RAC bajo condiciones ideales del cultivo, lo que corresponde al agua de evapotranspiración, definiéndose como el producto entre la ETo y el Kc [17], se calculó en valores decadiarios (dec).

$$
E T C[m m / d i a]=\mathrm{Kc} * \mathrm{ETO}
$$

Kc: Coeficiente del cultivo o uso consuntivo del cultivo el cual varía en cada etapa fenológica del cultivo, ETo: evapotranspiración de referencia ( $\mathrm{mm} /$ día).

\section{- Estimación de la evapotranspiración (verde y azul) del cultivo (ETc)}

El modelo presenta el requerimiento de agua verde del cultivo como la precipitación efectiva y corresponde al agua proveniente de la precipitación retenida en la zona no saturada del suelo que queda a disposición de las plantas [20], [21]; el resultado se da en mm, razón por la cual es necesario convertir el valor expresado en $\mathrm{m}^{3} / \mathrm{ha}$, a lo cual se le denomina consumo de agua del cultivo $\left(\mathrm{UAC}_{\mathrm{verde}}\right)$.

ETVerde $=$ ETc - Req Riego

El requerimiento de agua azul o requerimiento de riego del cultivo $\left(\mathrm{UAC}_{\mathrm{azul}}\right)$ se obtiene mediante la diferencia entre el requerimiento de agua del cultivo y la precipitación efectiva, de acuerdo a las estimaciones del módulo de programación del software; si esta diferencia es menor o igual a cero se tiene que no hay HH azul. Estas variables son dadas por el modelo en $\mathrm{mm}$, por lo cual se sigue el mismo procedimiento descrito para $\mathrm{UAC}_{\text {verde }}$ para llegar a $\mathrm{m}^{3} / \mathrm{ha}$.

Req Riego = ETc - Peff $(8)$

Finalmente, la suma de ambas, ETazul y ETverde es igual a ETcultivo.

\section{2) cálculo de la huella hídrica gris $\left(H_{\text {Gris }}\right)$}

El concepto de huella hídrica gris se introdujo como un indicador del volumen de agua dulce necesario para diluir la carga contaminante total, generada a partir de un proceso de producción en particular [22], en este caso el cultivo de aguacate cv. Hass. Para su cálculo, se utilizó como referente la contaminación del agua por causa de aplicación de fertilizantes, específicamente de nitrógeno $(\mathrm{N})$, ya que el fósforo (P) aplicado como fertilizante que no es absorbido por la planta, generalmente se acumula en el suelo y solo una fracción muy pequeña va hacia las fuentes de agua superficiales y subterráneas [23]. En este estudio se asumió una tasa de lixiviación del $10 \%$ para el nitrógeno $(\mathrm{N})$ total [24], [25] y 0\% para el fosforo (P). Se calculó según la siguiente expresión:

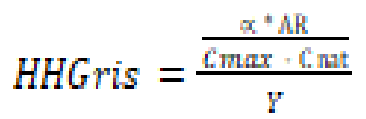

Teniendo en cuenta que para Colombia aún no existe una 
norma específica para Nitrógeno Total [26], se empleó una concentración máxima aceptable de $0.01 \mathrm{~kg} / \mathrm{m}^{3}$ de acuerdo con lo establecido en la normatividad colombiana [27], [28] para nitratos y nitritos, de igual manera una concentración natural igual cero [4]. La cantidad aplicada de productos químicos para el campo por hectárea (AR, $\mathrm{kg} / \mathrm{ha})$ se determinó en $150 \mathrm{Kg} / \mathrm{ha}$ de nitrógeno y un rendimiento del cultivo de 1,14 Ton/ha.

\section{RESULTADOS Y DISCUSIÓN}

\section{A. Diagnóstico del sistema productivo}

\section{1) Descripción de la unidad productiva}

Se empleó herramienta tecnológica Drone (DJI - FC220) y Software DroneDeploy con los que se estimó un rango de ubicación del cultivo entre los 1749.8 y 1850.9 msnm (Fig. 2). Igualmente se calculó el total de área sembrada en 21 ha de aguacate cv. Hass, divididas en 21 parcelas distribuidas heterogéneamente en el terreno, con densidad de siembra en promedio de 150 plantas/ha para un total de 3150 individuos. Las plantas presentaron una distancia de siembra de $6 \mathrm{~m} \times 6 \mathrm{~m}$ y una edad aproximada del cultivo de 5 años, los cuales produjeron en el periodo de interés ( 1 año) un total de 24 toneladas obteniendo un rendimiento de cultivo de 1.14 ton/ha.

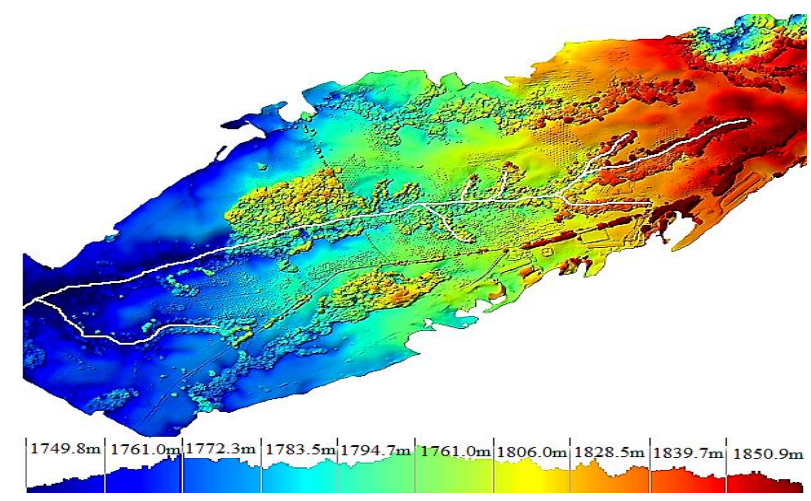

Fig. 2. Altura del área del cultivo de Aguacate cv. Hass. estimada con Software DroneDeploy

\section{2) información climática de las variables de interés}

Se tomó información de la estación climatológica Bremen dada su representatividad y cercanía a la unidad de estudio, localizada en el municipio de Filandia a $2.040 \mathrm{msnm}$, con coordenadas N $4^{\circ} 40^{\prime} 06^{\prime \prime}$ y W $75^{\circ} 36^{\prime} 01$ ' y pertenece a la red climatológica de la Corporación Autónoma regional del Quindío (CRQ). Se promediaron datos de las variables: temperatura máxima y mínima $\left({ }^{\circ} \mathrm{C}\right)$, humedad relativa $(\%)$, velocidad del viento $(\mathrm{m} / \mathrm{s})$, radiación (horas) y precipitación $(\mathrm{mm})$, con una resolución diaria de un periodo de datos intermitentes de aproximadamente nueve años.

De acuerdo con el promedio multianual de datos históricos reportados hasta abril de 2020, la zona de influencia de la estación Bremen presenta regímenes climatológicos bimodales con dos periodos de lluvia y dos periodos secos al año. Se reportan temperaturas máximas de $20.4{ }^{\circ} \mathrm{C}$ en el mes de agosto y temperaturas mínimas en el mes de octubre de $14.4^{\circ} \mathrm{C}$, una humedad relativa en un rango de $87,5-92.7 \%$, un brillo solar máximo de 5,6 horas y mínimo 1,75 horas en los meses de agosto y mayo respectivamente, finalmente una velocidad del viento que no supera los $2 \mathrm{~m} / \mathrm{s}$ a lo largo del año (ver Tabla I).

TABLA I.

PROMEDIO DE DATOS CLIMATOLOGICOS HISTORICOS DE LA ESTACIÓN BREMEN

\begin{tabular}{|l|c|c|c|c|c|c|c|c|c|c|c|c|}
\hline $\begin{array}{l}\text { VARIABLE / } \\
\text { MES }\end{array}$ & ENE & FEB & MAR & ABR & MAY & JUN & JUL & AGO & SEP & OCT & NOV & DIC \\
\hline $\begin{array}{l}\text { Brillo solar } \\
\text { (Horas) }\end{array}$ & 3,7 & 3,3 & 2,3 & 1,8 & 1,75 & 2,9 & 4,52 & 5,6 & 3,9 & 2,5 & 2,1 & 2,4 \\
\hline $\begin{array}{l}\text { Temperatura } \\
\text { Min. }\left({ }^{\circ} \mathbf{C}\right)\end{array}$ & 15,3 & 15,3 & 15,1 & 14,6 & 15,3 & 14,9 & 15,5 & 15,7 & 14,7 & 14,4 & 14,8 & 14,8 \\
\hline $\begin{array}{l}\text { Temperatura } \\
\text { Max. }\left({ }^{\circ} \mathbf{C}\right)\end{array}$ & 18,8 & 18,9 & 18,9 & 19,2 & 19,3 & 19,1 & 20,2 & 20,4 & 20,3 & 18,7 & 18,3 & 19,3 \\
\hline $\begin{array}{l}\text { Humedad } \\
\text { relativa (\%) }\end{array}$ & 92,7 & 92,7 & 90,4 & 87,5 & 89,5 & 89,7 & 91,3 & 90,7 & 91,6 & 89,8 & 88,8 & 89,1 \\
\hline $\begin{array}{l}\text { Velocidad del } \\
\text { viento (m/s) }\end{array}$ & 1,39 & 1,38 & 1,27 & 1,57 & 1,47 & 1,59 & 1,55 & 1,96 & 1,14 & 1,43 & 1,41 & 1,43 \\
\hline
\end{tabular}

3) fertilización y aporte de nitrógeno al cultivo de aguacate cv. Hass.

El proceso de fertilización del Cultivo de aguate cv. Has en la unidad de estudio, es llevado a cabo por aplicaciones edáficas mensuales de diferentes nutrientes, de las cuales el $8 \%$ corresponde a nitrógeno $(\mathrm{N})$ por cada 45 kilogramos producto. Lo anterior se distribuye en $150 \mathrm{kgN} / \mathrm{ha} / \mathrm{año}$, donde cada individuo de aguacate cv. Hass recibió aproximadamente 1.000 grN/año, lo que concuerda con recomendaciones de [29].

4) Comportamiento productivo del cultivo de aguacate cv. Hass en la unidad de estudio.

Se obtuvo una caracterización preliminar del comportamiento productivo general del cultivo, definiendo las principales etapas fenológicas en los meses que tienen lugar dos ciclos de producción de aguacate Hass, como se muestra en el siguiente calendario fenológico de la tabla II.

TABLA II.

CALENDARIO FENOLÓGICO DE AGUACATE HASS EN LA UNIDAD DE ESTUDIO

\begin{tabular}{|c|c|c|c|c|c|c|c|c|c|c|c|c|}
\hline Etapa Fenológica/Mes & E & F & M & A & M & J & J & A & S & O & N & D \\
\hline Floración & & & & & & & & & & & & \\
\hline Formación - fruto & & & & & & & & & & & & \\
\hline Cosecha & & & & & & & & & & & & \\
\hline
\end{tabular}

El cultivo presentó dos flujos vegetativos y dos flujos florales en el periodo de análisis correspondientes a dos cosechas ( 1 cada 6 meses), la primera en el mes de octubre de 2019 y en la segunda en el mes de abril de 2020.

\section{B. Huella hídrica del cultivo de aguacate cv. Hass Persea americana Mill.}


1) Cálculo de la evapotranspiración (ET $T_{O}$ y determinación del coeficiente de cultivo $(\mathrm{Kc})$

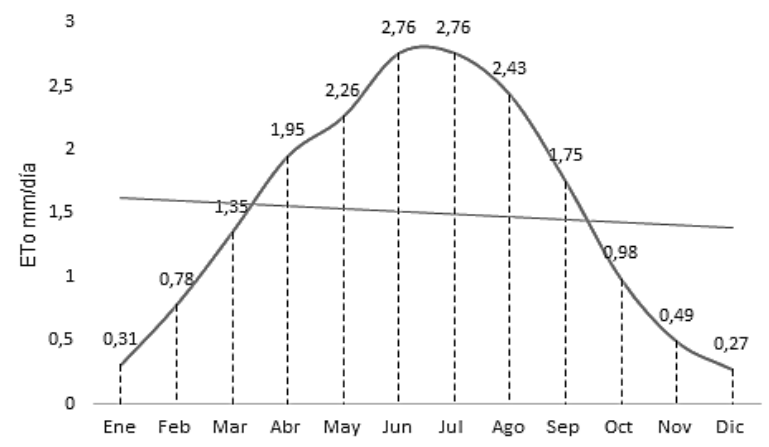

Fig. 3. Evapotranspiración de referencia mensual (mm/día) estimada por el software Cropwat 8.0

De acuerdo con los cálculos de Cropwat 8.0 (Fig. 3), la evapotranspiración de referencia $\left(\mathrm{ET}_{\mathrm{O}}\right)$ muestra datos ascendentes en el primer periodo del año, con lo cual el crecimiento de la curva se detiene en los meses de junio y julio, quienes reportan el mismo valor $(2,76 \mathrm{~mm} / \mathrm{día})$ siendo los más altos; a partir de allí, los valores descienden en el segundo semestre, siendo el mes de diciembre quien reporta el valor más bajo con $0.27 \mathrm{~mm} /$ día, seguido al mes de enero reportando $0.31 \mathrm{~mm} /$ día. Finalmente, la estimación media anual de $\mathrm{ET}_{\mathrm{O}}$ fue de $1.51 \mathrm{~mm} /$ día.

El valor del coeficiente de cultivo $\mathrm{Kc}$, no se conoce para aguacate var. Hass en condiciones edafoclimaticas de Colombia, sin embargo, para el presente proyecto se trabajó siguiendo la recomendación de [17], quien menciona que, bajo condiciones andinas en la etapa de desarrollo media, puede estimarse en un coeficiente del cultivo $(\mathrm{Kc})$ igual a 0.75 , similar a lo reportado por diferentes estudios en América [16], [30], [31], [32] - [33].

2) Comparación, análisis de sostenibilidad ambiental y determinación de la huella del cultivo de aguacate cv. Hass del DCS BB.

El área de influencia del DCS BB reporta precipitaciones medias anuales de $3044.4 \mathrm{~mm} / \mathrm{año}$, lo que supera ampliamente el rango sugerido por [34], quien menciona que la variedad Hass requiere entre 1200 a $1800 \mathrm{~mm}$ de lluvia anual. La precipitación efectiva establece que en promedio $1681.2 \mathrm{~mm}$ de lluvia quedan a disposición de las plantas de aguacate cv. Hass para su aprovechamiento anual, lo que corresponde al $55.2 \%$ del total de precipitaciones reportadas.

La información climática obtenida en una estación con diferencia de $250 \mathrm{msnm}$ con respecto al punto de estudio no presenta alteraciones y sus valores se mantienen de forma similar.

De acuerdo con los registros de las condiciones climáticas históricas reportadas por la estación Bremen, los valores de la precipitación efectiva y por consiguiente de la precipitación media superan la evapotranspiración del cultivo durante el ciclo. Julio y agosto se muestran como los meses donde el volumen de las precipitaciones es más cercano a los requerimientos hídricos del cultivo, esto sin que se presenten déficits del recurso. El mes de precipitaciones más altas en el primer semestre del año fue abril con $292,3 \mathrm{~mm}$ de lluvia en promedio y en la segunda parte del año sobresale notoriamente el mes de noviembre reportando $438,2 \mathrm{~mm}$, siendo el mes con el valor promedio más alto.

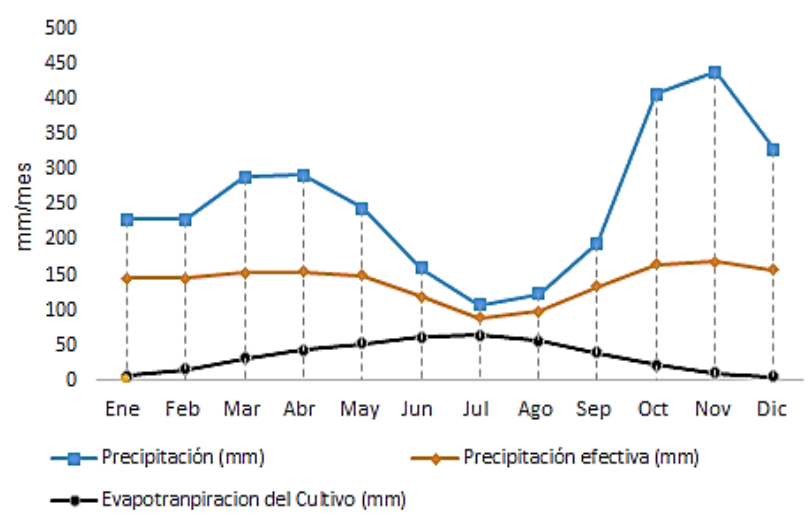

Fig. 4. Balance mensual entre la precipitación, precipitación efectiva y la evapotranspiración del cultivo de aguacate cv. Hass en el DCC BB.

La Fig. 4 muestra que el aporte hídrico natural es bastante alto con respecto a las perdidas hídricas del cultivo por evapotranspiración, las cuales se determinaron en 413,9 mm durante el periodo de estudio, estas suplidas en su totalidad por la precipitación efectiva $(1681,2 \mathrm{~mm})$, quien pone a disposición del cultivo 3 veces más de lo requerido. De acuerdo con lo anterior, el valor de huella hídrica verde estimado para el cultivo de aguacate cv. Hass localizado en área de influencia del Distrito de conservación de suelos se estimó en $3630 \mathrm{~m}^{3} / \mathrm{ton}$.

Dado que el resultado de la precipitación efectiva es mayor a la evapotranspiración del cultivo (8), el valor de los requerimientos de riego fue estimado en cero, lo cual indica un componente de huella hídrica azul con el mismo valor $(0,0$ $\mathrm{m}^{3} / \mathrm{ton}$ ), indicando que durante el periodo de tiempo evaluado no fue necesaria la presión sobre las corrientes hídricas circunvecinas para su uso consuntivo. La huella hídrica azul es insostenible cuando excede el agua azul renovable disponible, violando así el estándar de flujo ambiental [35], lo cual no se presentó en el periodo evaluado indicando un aspecto a favor de la sostenibilidad ambiental en general, especialmente de las dinámicas de las comunidades biológicas que desarrollan sus ciclos de vida en los ecosistemas acuáticos presentes en el área de influencia; también garantiza la sostenibilidad del desarrollo de la agricultura en términos de disponibilidad y acceso al recurso, ya que por parte del cultivo en estudio se mantienen los caudales de los cuerpos de agua de forma natural.

El valor obtenido para el componente gris de la huella hídrica del cultivo de aguacate cv. Hass en el DCS BB fue de $1315 \mathrm{~m}^{3} /$ ton, indicando la cantidad de agua que el cuerpo receptor necesita para diluir la fracción de lixiviación y escorrentía que llega, en este caso del nitrógeno antropogénico 
(N) producto del proceso de fertilización inorgánico que se llevó a cabo en la unidad de estudio. Cabe resaltar que la estimación de la huella gris del presente trabajo se enfocó en la fracción de nitrógeno $(\mathrm{N})$, excluyendo otros elementos fertilizantes además de sustancias inmersas en pesticidas aplicados en el ciclo evaluado, lo que posiblemente aumentaría el valor de este componente y por consiguiente la huella hídrica. Lo anterior teniendo en cuenta que los plaguicidas llegan de igual manera a las fuentes hídricas a través de la escorrentía subterránea y superficial del agua de riego y de lluvia, y a través de su lixiviación cerca del agua de percolación, siendo así una fuente de contaminación difusa del agua dulce [36].

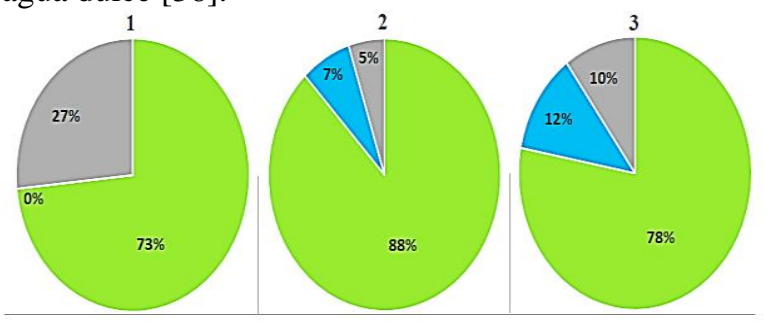

Fig. 5. Comparación de porcentajes de huella hídrica verde, azul y gris entre cultivo de aguacate cv. Hass en el DCS BB (1) (73\% verde, $0 \%$ azul y $27 \%$ gris), huella hídrica agrícola de Colombia (2) y huella hídrica agrícola mundial (3).

Las proporciones de huella hídrica del cultivo de aguacate cv. Hass del DCS BB (73\% verde, $0 \%$ azul y $27 \%$ gris) Fig. 5, se comportan en ciertos aspectos de manera similar con las tendencias agrícolas nacional y mundial, teniendo en cuenta que los modelos y supuestos para los respectivos cálculos fueron diferentes, existe una concordancia en la predominancia del componente verde. Para el sector agrícola colombiano se estimaron valores de huella hídrica distribuidos en: $88 \%$ verde, $7 \%$ azul y el restante $5 \%$ gris [2], a nivel global Mekonnen y Hoekstra reportan una huella hídrica relacionada con la producción agrícola para el período 1996 2005, de 7404 mil millones de metros cúbicos por año correspondiente a: $78 \%$ verde, $12 \%$ azul, $10 \%$ gris [37].

La huella hídrica azul para el aguacate en Colombia se estimó en 28,3 millones de $\mathrm{m}^{3} / \mathrm{año}$ [38], sin embargo, el cultivo de aguacate cv. Hass en estudio no aporta a este indicador, debido a que las condiciones ambientales y climatológicas de la zona cumplieron satisfactoriamente los requerimientos hídricos del cultivo. El componente gris nacional y mundial reporta valores por debajo de lo determinado por el presente trabajo, este elevado valor indica un alto riesgo a la sostenibilidad de los recursos hídricos de esta zona de la reserva natural, para esto es importante continuar con estudios tendientes a determinar la capacidad que tiene la cuenca hidrográfica en jurisdicción del DCS BB, de asimilar (diluir) no solo las fracciones de agroquímicos que llegan a las corrientes por lixiviación y escorrentía, sino también de las diferentes unidades de producción en toda el área.

Mekonnen y Hoekstra estimaron la huella hídrica media mundial del cultivo de aguacate en general reportando un valor de $1981 \mathrm{~m}^{3} /$ ton [36], donde los componentes más elevados fueron el verde y el gris con el mismo valor (849 $\mathrm{m}^{3} /$ ton), seguido de la huella hídrica azul estimado en 283 $\mathrm{m}^{3} /$ ton. Al comparar estos valores con los obtenidos en el presente estudio para aguacate $\mathrm{cv}$. Hass, se puede marcar una coincidencia en la predominancia entre los componentes verde y gris, pero con valores mucho más altos; el componente verde es aproximadamente 4,27 veces el valor de la media mundial, mientras que el componente gris reporta una diferencia de 466 $\mathrm{m}^{3} /$ ton más del recurso hídrico y finalmente el componente de huella hídrica azul es el único por debajo de los valores comparados al ser reportado en cero.

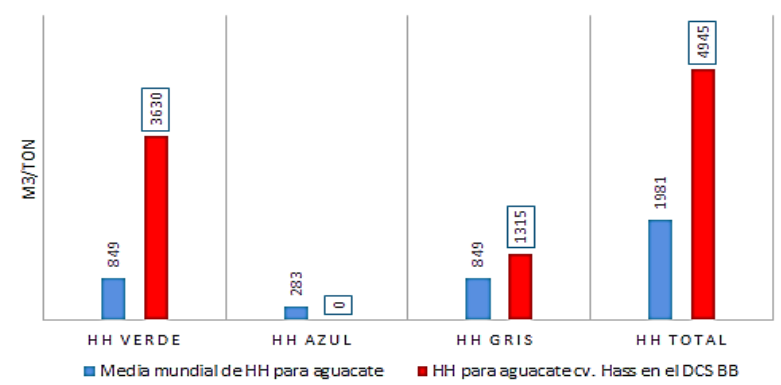

Fig. 6. Comparación entre componentes de huella hídrica obtenida para el cultivo de aguacate cv. Hass en el DCS BB y promedio mundial para aguacate calculado Water Footprint Network WFP.

La huella hídrica total del cultivo en estudio se determinó en $4945 \mathrm{~m}^{3} /$ ton (Fig. 6), siendo un valor bastante alto equivalente a 2,5 veces la media mundial. Desde el punto de vista del agua virtual, entendida como el agua "contenida" en el producto, no únicamente la cantidad contenida físicamente en el mismo, sino la cantidad de agua utilizada en este caso por el cultivo para la generación del mismo [39], se necesitan 4945 litros de agua para generar un kilogramo de aguacate cv. Hass producido en la unidad de estudio ubicada en el DCS BB.

El rendimiento de cultivo influyo directamente sobre la determinación de la huella hídrica, dado que los cultivos con un alto rendimiento o biomasa cosechada generalmente tienen una huella hídrica más pequeña por tonelada que los cultivos con un rendimiento bajo [37]. En promedio el departamento del Quindío registra un rendimiento de cultivo de aguacate cv. Hass de 8,5 aproximadamente [40], superando ampliamente el rendimiento del cultivo objeto de estudio el cual se estimó en $1.14 \mathrm{Ton} / \mathrm{ha}$.

\section{CONCLUSIONES}

El aporte natural de agua por medio de las precipitaciones en área de influencia del Distrito de Conservación de Suelos Barbas Bremen, cubrió eficientemente las necesidades hídricas del cultivo de aguacate cv. Hass durante los dos ciclos de producción evaluados, esto hace que el componente de huella hídrica verde sea el valor más alto de los tres que se estimaron.

El uso de agua verde se consideró un indicador sostenible ya que los requerimientos hídricos del cultivo estuvieron muy por debajo de la disponibilidad del recurso a causa del aporte de las precipitaciones. 
Siendo el Departamento del Quindío uno de los principales productores de aguacate cv. Has de Colombia, es importante contar con estimaciones de su huella hídrica como una herramienta complementaria para la evaluación integral de la sostenibilidad ambiental, aun con más énfasis si estos se ubican en áreas de protección e importancia ecológica.

La presión antrópica sobre las corrientes hídricas aledañas al cultivo fue nula desde el punto de vista de la necesidad de riego, indicando un aporte positivo sostenibilidad ambiental de los recursos hídricos en la zona.

El bajo rendimiento del cultivo de aguacate cv. Hass del DCS BB influye directamente en el alto valor de la huella hídrica estimada y por consecuencia en la huella hídrica total, aumentar esta variable permitirá aprovechar de mejor forma el recurso agua.

Virtualmente se necesitan 4945 litros de agua producir un kilogramo de aguacate cv. Hass en la unidad de estudio, valor que sobre pasa el promedio mundial para la producción de aguacate en general.

\section{REFERENCIAS}

[1] IDEAM - Instituto Colombiano de Hidrología, Meteorología y Estudios Ambientales (2019). Estudio nacional del agua 2018. Bogotá: Ideam, $452 \mathrm{pp}$.

[2] Arévalo, D. (2012). Una mirada a la agricultura de Colombia desde su huella hídrica. WWF - Reporte para-Colombia. 48 pp.

[3] FAO - Food and Agriculture Organization of the United Nations (2013). Una contribución a la seguridad alimentaria y la adaptación al cambio climático. Asociación mundial para el agua, capítulo centroamérica organización de las naciones unidas para la alimentación y la agricultura. Tegucigalpa, Honduras, $72 \mathrm{pp}$.

[4] Hoekstra, A., Chapagain, A., Aldaya, M., \& Mekonnen, M. The Water Footprint Assessment Manual - Setting the Global Standard. Publishing for a sustainable future. London and Washington, DC. ISBN: 978-1-84971-279-8 hardback 2011.

[5] Hoekstra, A. Y. The water footprint of modern consumer society. London, UK: Routledge. First edition published by Routledge 2013.

[6] Mekonnen, M.M., Hoekstra, A.Y,. Global gray water footprint and water pollution levels related to anthropogenic nitrogen loads to fresh water. Environmental Science and Technology, 49(21): 12860 - 12868, 2015

[7] Van Miegrot, H y Johnsson, D.W. Feedbacks and synergism among biochemistry, basic ecology, and forest soil science. Forest Ecology an-Management 258: 2214-23, 2009.

[8] Ministerio de Agricultura y de Desarrollo Rural (2018). Cadena de aguacate, indicadores e instrumentos. Dirección cadenas agrícolas y forestales. Agosto, Bogotá - Colombia. 21 pp.

[9] Ministerio de Agricultura (2019). Colombia se convierte en productor de aguacate Hass de clase mundial, boletín página virtual - el campo es de todos. Enero, Bogotá - Colombia.

[10] ANALDEX - Asociación nacional de comercio exterior (2020). Colombia aumenta sus exportaciones de aguacate Hass a China. Noticia de portafolio - página Web.

[11] Anguiano, J., Coria, V., Ruiz., J., Chávez, G., \& Alcántar., J. Caracterización edáfica y climática del área productora de aguacate Persea americana cv. Hass, Procc. V World Congress en Michoacán, México. 323-328 pp, 2003.

[12] Gavito, M., Calderón, M., Martinez, J., Ayala, R., \& Ramírez, E. Evaluación del impacto ecológico del cultivo de aguacate a nivel regional y de parcela en el Estado de Michoacán: validación de indicadores ambientales en los principales tipos de producción. Informe final. Michoacán, México. 85 pp, 2012.

[13] CRQ - Corporación Autónoma Regional del Quindío (2014). Plan de manejo del distrito de conservación de suelos Barbas-Bremen, jurisdicción del departamento del Quindío. Fondo de compensación ambiental (fca) del Ministerio de ambiente y desarrollo sostenible. Armenia, Colombia. 209 pp.

[14] Universidad del Cauca (2018). Guía para la caracterización de unidades de producción agropecuaria. Sistemas integrados de producción agropecuaria I. Facultad de ciencias agropecuarias - ing. Agropecuaria. Cauca, Colombia. 23 pp.

[15] Betancourt M., Posada V., Lastra C., Carrillo L., y Montealegre V. (2017). Informe final. Carta Acuerdo FAO-ANZORC. Bogotá D. C., Colombia. 420 pp.

[16] FAO - Food and Agriculture Organization of the United Nations (2006). Evapotranspiración del cultivo: Guías para la determinación de los requerimientos de agua de los cultivos. Estudio FAO riego y drenaje. Rome, paper 56. 322 pp.

[17] Grajales, J. Uso racional del agua de riego en cultivos de aguacate hass (Persea americana) en tres zonas productoras de Colombia. Tesis Universidad Nacional de Colombia. Cede: Palmira - Colombia, 2017.

[18] FAO - Food and Agriculture Organization of the United Nations (2010). CROPWAT 8.0 Model, Food and Agriculture Organizationof the United Nations, Rome, Italy.

[19] Doorenbos, J. and W. O. Pruitt, W. (1977). Crop Water Requirements. FAO Irrig. And Drain. Paper 24, Food and Agriculture Organization of the United Nations, Rome. $156 \mathrm{pp}$.

[20] Hoekstra, A. Y (2013). The Water Footprint of Modern Consumer Society. London: Routledge, soft and Hardcover. Edit Earthscan Routledge - 224 pp.

[21] Vanham, D., \& Bidoglio, G. A Review on the Indicador Water Footprint for the EU28. Ecological Indicators, 26, 61 - 75 pp. 2013.

[22] Hoekstra AY, Chapagain AK. Huellas hídricas de las naciones: uso del agua por parte de las personas en función de su patrón de consumo Recurso de agua. Manag. , 21, 35 - 48 pp.,2006.

[23] Ercin, A. E; Aldaya, M. \& Hoekstra, A.Y. (2011). The water footprint of soy milk and soy burger and equivalent animal products. Value of Water: Research Report Series N. 49. UNESCO - IHE. Paris, France.

[24] Hoekstra, A. Y. \& Chapagain, A. K. (2008). Globalization of Water: Sharing the Planet'S Freshwater Resources; Blackwell: Oxford.

[25] Ovalle, M. J. (2012). Estimación de la huella hídrica de cultivos con potencial bioenergético en la provincia de Limarí, región de Coquimbo. Chile. Santiago de chile. Tesis. 54 pp.

[26] IDEAM - Instituto Colombiano de Hidrología, Meteorología y Estudios Ambientales (2007). Ficha técnica nitrógeno total en agua por el método semi-micro kjeldahl - electrodo de amoniaco. Versión 2.

[27] Ministerio de Agricultura (MA). (1984). Decreto 1594 de junio de 1984. Por el cual se reglamenta parcialmente el Título I de la Ley 9 de 1979, Bogotá: Ministerio de Agricultura. Bogotá.

[28] Ministerio de la Protección Social y Ministerio de Ambiente, Vivienda y Desarrollo Territorial. (2007). Por medio de la cual se señalan características, instrumentos básicos y frecuencias del sistema de control y vigilancia para la calidad del agua para consumo humano. Bogotá: MPS y MAVDT, 2007. (Resolución 2115 de 2007).

[29] Bernal, J. A. y Díaz, C. A. (2005) Manual técnico No. 5: Tecnología para el cultivo del Aguacate. Colombia: Ed. Produmedios. Corporación Colombiana de Investigación Agropecuaria CORPOICA, Centro de Investigación La Selva, Río Negro, Antioquia.

[30] Chuchon, E. (2015). Calculo de demanda de agua y necesidad de riego para cultivo anual, forraje y frutal Con aplicación de Cropwat $8.0-$ FAO - Tambillo (3250m.s.n.m.) - Ayacucho. V Congreso Nacional-IV Congreso Iberoamericano de riego y drenaje, Lima, Perú.

[31] Ferreyra R, Selles G, Gil P, Ruiz R, Barrera C, Maldonado P, Celedón J. (2007). Manejo del riego y suelo en palto. Boletín del Instituto de Investigaciones Agropecuarias. Gobierno de Chile. 160:1-123 pp.

[32] Faber, B. Avocado irrigation in California. Presentado en seminario: Manejo del riego y suelo del cultivo del palto, INIA, La Cruz, Chile, 27-28 de septiembre de 2006, $5 \mathrm{pp}$.

[33] Gardiazabal, F., Magdahl, C., Mena, F., Wilhelmy, C. (2003). Determinación del coeficiente del cultivo $(\mathrm{Kc})$ para paltos cv. Hass en Chile. Proceedings V World Avocado Congress (Actas V Congreso Mundial del Aguacate). 329-334 pp.

[34] Bartoli, J.A. Manual técnico del cultivo del aguacate Hass (Persea americana L.). La Lima, Honduras: Fundación Hondureña de Investigación, 2008.

[35] Mekonnen, M.M., Hoekstra, A.Y. (2020). Sustainability of the blue water footprint of crops. Advances in water resourses 143 (2020) 103639.

[36] N. Sasakova, G. Gregova, D. Takacova, J. Mojzisova, I. Papajova, J. Venglovsky, T. Szaboova , S. Kovacova Contaminación de aguas superficiales y subterráneas por fuentes relacionadas con actividades agrícolas Frente. Sostener. Food Syst., 2 (2018) , 1 - 11 pp. 
[37] Mekonnen, M. M., \& Hoekstra, A. Y. (2010). The green, Blue and Grey Water Footprint of Crops and Derived Crops Products Value of Water Research Report Series No.47, UNESCO-IHE, Delft. 24 pp.

[38] CTA, GSI-LAC, COSUDE, IDEAM, (2015). Evaluación Multisectorial de la Huella Hídrica en Colombia. Resultados por subzonas hidrográficas en el marco del Estudio Nacional del Agua 2014. Medellín, Colombia.

[39] Allan, T. Virtual water: Tackling the threat to our planet's most precious resourse. London: I. B. Tauris. Vol. 36, No. 7, November 2011, 948-950, 2011.

[40] Ministerio de Agricultura y Desarrollo Rural (2018). Evaluaciones agropecuarias municipales EVA. Base histórica de periodo 2007 2018. Producción nacional agrícola, Bogotá.

Jhon Faber Naranjo Pardo es Licenciado en Biología y Educación Ambiental de la Universidad del Quindío, Magister en Desarrollo Sostenible y Medio Ambiente de la Universidad de Manizales. Actualmente es contratista independiente, ha realizado trabajos de investigación en calidad ecológica en ríos andinos y ha participado como ponente en simposios de trabajos de investigación.

Henry Reyes Pineda es ingeniero Químico egresado de la Universidad Nacional de Colombia, Sede Manizales con Especialización en Educación Ambiental, Especialización en Ingeniería Electroquímica y Corrosión, Diploma de Estudios Avanzados en Tecnologías de Membranas, Electroquímica y medio Ambiente Seguridad Nuclear y con un Doctorado en Ingeniería Química y Nuclear de la Universidad Politécnica de Valencia, España. Actualmente es profesor de tiempo completo de la Universidad del Quindío, Armenia, Colombia y Decano de la Facultad de Ciencias Agroindustriales; es docente de la Maestría en Desarrollo Sostenible y Medio Ambiente de la Universidad de Manizales; hace parte del Grupo de Investigación en Ciencias Ambientales, INCIAM y Grupo de Investigación del CIMAD. Entre sus áreas de trabajo investigativo se encuentran La Ingeniería Electroquímica, Los Procesos ambientales, el Tratamiento de residuos sólidos y líquidos, el Diseño de Reactores, entre otros. El Doctor Reyes Pineda, se gradúo en el 2007, obteniendo la máxima calificación en su Tesis Doctoral: "Cum Laude" Ha sido profesor visitante en tres oportunidades a la Universidad Politécnica de Valencia, España y en dos ocasiones a la Universidad de santa Cruz del Sur, Brasil, desarrollando actividades académicas e investigativas. Igualmente ha participado como conferencista en diferentes congresos nacionales e internacionales. 